\title{
Lung Development and Notch Signaling
}

\author{
Mitsuru Morimoto
}

\begin{abstract}
The respiratory system is connected to the cardiovascular system physically and physiologically. This chapter overviews the morphogenesis and epithelial development of the lung. The airway branching structure is formed as development progresses and is controlled by reciprocal mesenchymal-epithelial interactions. During the branching process, the distal terminal buds are thought to contain a population of multipotent epithelial progenitors that are more proliferative than proximal cells. This predominant proliferation in the distal tip leads to the lung bud extension toward the distal end. As the bronchial tree extends further, descendants of these multipotent cells give rise to lineage-restricted progenitors in the conducting airways. Notch signaling is used repeatedly to organize three epithelial cell types: Club, ciliated, and neuroendocrine (NE) cells. The Notch-mediated fate selection of Club/ciliated cells and of the size of NE cell clusters is regulated by different mechanisms. The Club/ciliated cell fate decision is mediated exclusively by Notch 2 in response to Jag1. In contrast, all three Notch receptors contribute to robustly regulate the NE cell-cluster size. High-resolution whole-mount imaging of the developing lung revealed that the NE cell cluster appears at the stereotypic positions at the bifurcating area of the branching airways. Moreover, the 4D imaging, 3D plus live-imaging, method for developing lung epithelial cells discovered dynamics of NE cell clustering in which NE cells appear at inter-bifurcation area as solitary cells and migrate toward the branching points to form clusters. Further analyses determined that Notch signaling regulates the number of solitary NE cells in a lateral-inhibition fashion.
\end{abstract}

\footnotetext{
M. Morimoto $(\bowtie)$

RIKEN Center for Biosystems Dynamics Research, Laboratory for Lung Development and Regeneration, Kobe, Japan

e-mail: mitsuru.morimoto@ riken.jp 


\section{Keywords}

Lung $\cdot$ Notch signaling $\cdot$ Cell fate selection $\cdot$ Live imaging $\cdot$ Directional cell migration

\subsection{Introduction}

The lung facilitates gas exchange by maximizing ventilation efficiency and the surface area of interface to the capillaries. The developing lung forms an intricate, but stereotypic, branching airway structure and arrayed epithelial cells (Fig. 3.1). Air from the external environment includes viruses, bacteria, and toxic chemical compounds, which means that the airways may become damaged and need to be repaired rapidly. For these reasons, the lung is a useful model organ for studying cellular events that are conserved between organogenesis in the embryo and tissue regeneration in the adult.

\subsection{Morphogenesis and Epithelial Progenitors}

For the last two decades, extensive studies using mouse models have revealed biological processes and genes that regulate lung development. Although there are significant differences in tissue size, the lungs in human and mouse display similar

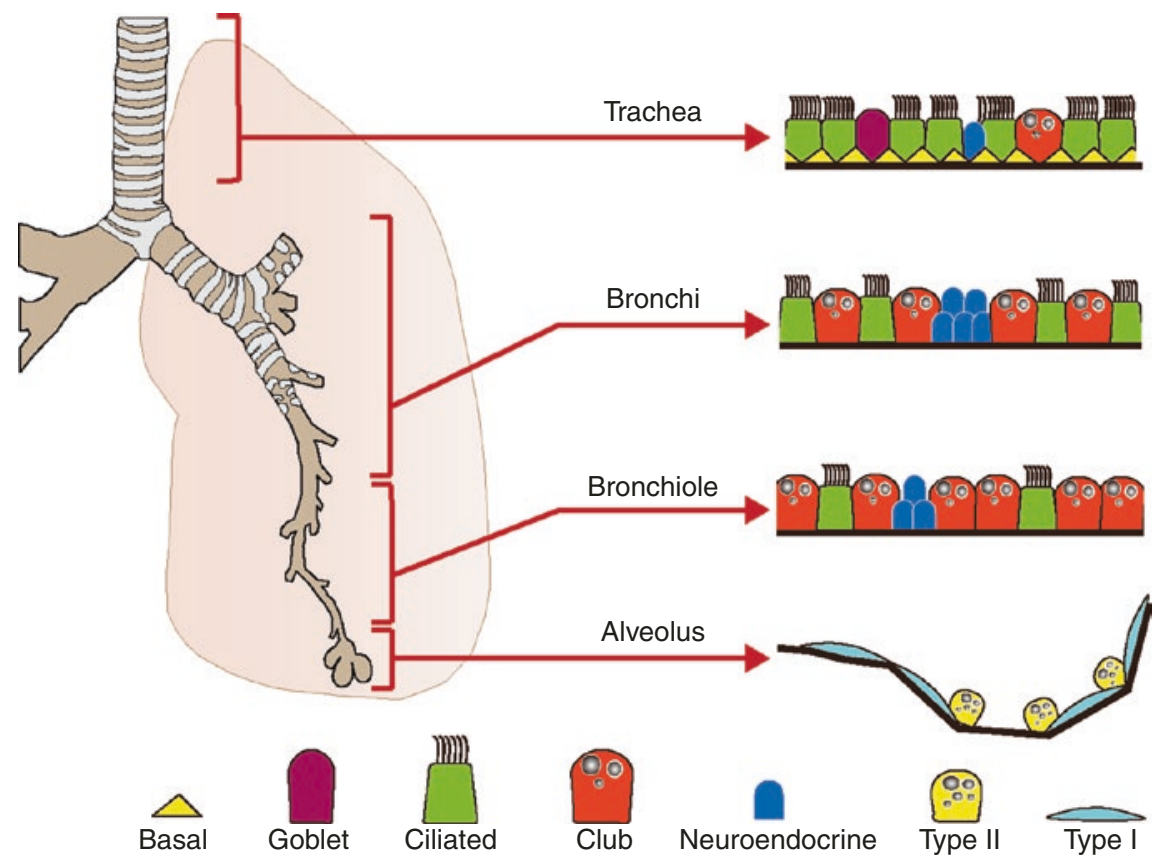

Fig. 3.1 The structure of the lung airways and their epithelial components 
Fig. 3.2 The distal tip stem cells and proximal differentiated cells in developing airways during pseudoglandular stage

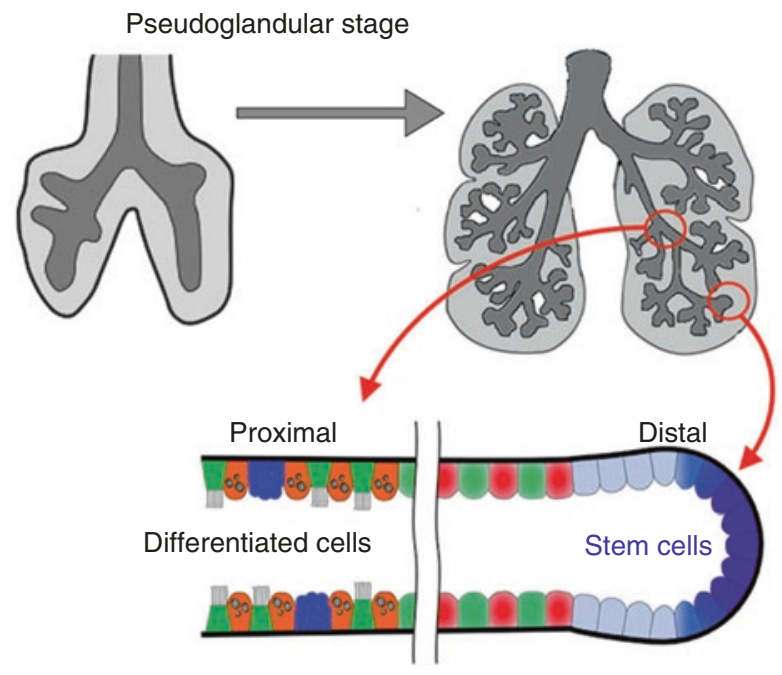

histology and pathology [1], suggesting that the mouse lung may be a useful model system for investigating organ biology and human disease. Lung development relies on reciprocal mesenchymal-epithelial interactions orchestrated by temporal and spatial expression waves of multiple secreted factors and their downstream effectors [2]. Airway branching morphogenesis takes place at E11.5-16.5 in the mouse and at 5-17 weeks in human embryo (known as pseudoglandular stage, during which most of airway branching morphogenesis takes place); it is thought that the terminal buds contain a population of multipotent epithelial progenitors (Fig. 3.2) [3]. While the buds extend and branch in a genetically coordinated stereotypic pattern [4], the progenitors give rise to lineage-restricted descendants that produce at least seven major cell types in the 'stalk' region $[3,5]$. Thus, the early stalks form the proximal airway, then the distal airways, and finally the alveoli [6]. In the proximal airway, the tracheal epithelium consists of the basal, goblet, Club (secretory), and ciliated cells. Smaller bronchi contain the latter two cell types and pulmonary neuroendocrine (NE) cells. The distal-most airway, the alveolus, is lined with thin layers of flat Type I cells and cuboidal Type II cells (Fig. 3.1).

\subsection{Notch Signaling Controls Both Epithelial Cell Fates and Distributions}

One of the outstanding questions in the field is how epithelial cells interact and exchange information to generate and maintain the appropriate balance in their respective cell numbers and distributions. Using genetic engineering methods, we have performed stepwise removal of the components of Notch signaling, a key signaling in cell fate decisions in developing lung epithelium, and revealed that Notch signaling is used reiteratively to organize three major epithelial cell types: Club, 
a

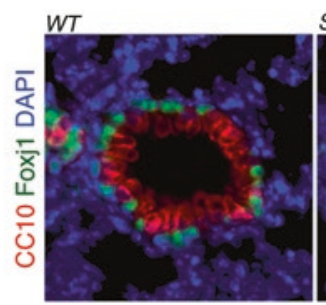

SHH-Cre, $\mathrm{N}^{\text {th }}$ SHH-Cre, $\mathrm{N2}^{\prime \prime}$
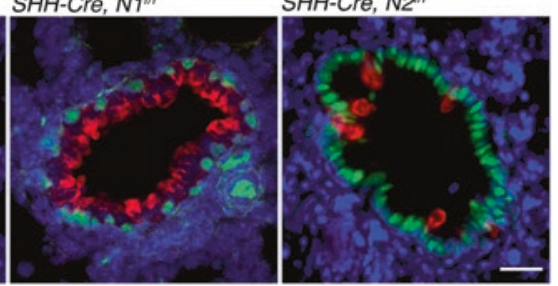

b

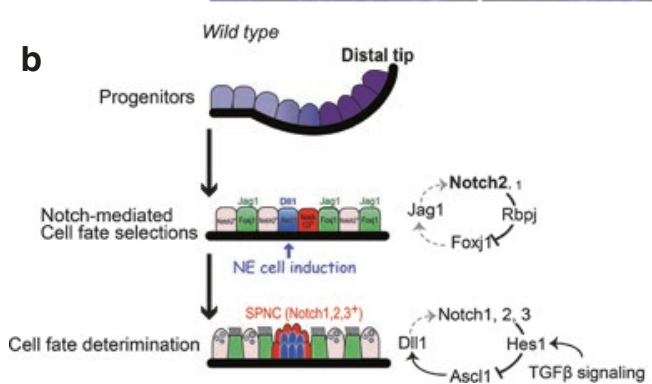

$\mathrm{SHH}-\mathrm{Cre}, \mathrm{N} 2^{\prime \prime}$

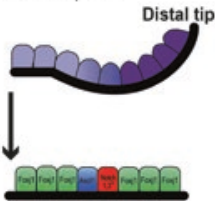

$\mathrm{SHH}-\mathrm{Cre}, \mathrm{N} 1^{n \pi}, \mathrm{N} 2^{2 n}, \mathrm{~N} 3^{-}$
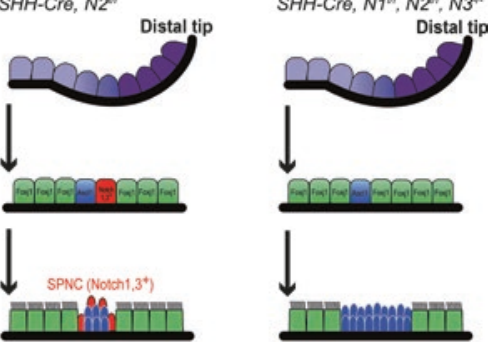

Fig. 3.3 Loss of Notch signaling phenotypes within airway epithelium. (a) Immunostainings for Club (CC10, Red) and ciliated (Foxj1, Green) cells of epithelial Notch1 or 2 conditional KO lung. (b) Schematic diagrams of the proposed regulatory mechanisms involved in Notch-mediated cell fate determination during bronchial epithelial development. In the pseudoglandular stage, epithelial progenitors (purple) located at the distal tip produce descendants that differentiate into Club (pink), ciliated (green), SPNC (red) and NE (blue) cells

ciliated, and NE cells [7, 8]. We also identified significant differences between the selection of Club/ciliated cells and the size regulation of NE cell clusters. The Club/ ciliated cell fate decision is mediated exclusively by Notch2 in response to Jag1 with negligible contributions from Notch1 and 3 (Fig. 3.3a). In contrast, all three Notch receptors respond to Dll1 and contribute in an additive manner to regulate NE cell cluster numbers and size (Fig. 3.3b). These results indicate that two distinct Notch signaling pathways, involving Jag1-Notch2 and Dll1-Notch123 signaling, respectively, coordinate the number and distribution of the major epithelial cell types of the conducting airway in lung organogenesis. However, at the time we were unable to clarify the Notch-mediated mechanism by which the NE cell number and cluster size are regulated.

\subsection{Development of NE Cell Clusters on Bifurcating Area of Branching Airways}

The pulmonary NE cells are thought to function as chemoreceptors and as a component of the stem cell niche and are also the cells of origin in small-cell lung cancer. NE cells often localize at bifurcation points of airway tubes, forming small clusters called neuroepithelial bodies (NEBs). These are referred to as "nodal" NEBs [9] whereas NEBs in inter-bifurcation regions are called "internodal" (Fig. 3.4). Despite 
Fig. 3.4 Immu-

nohistochemistry of the NE cell marker CGRP (brown) for E18.5 mouse lung. The arrowheads indicate the NEBs on bifurcation points (black) or inter-bifurcation region (gray)

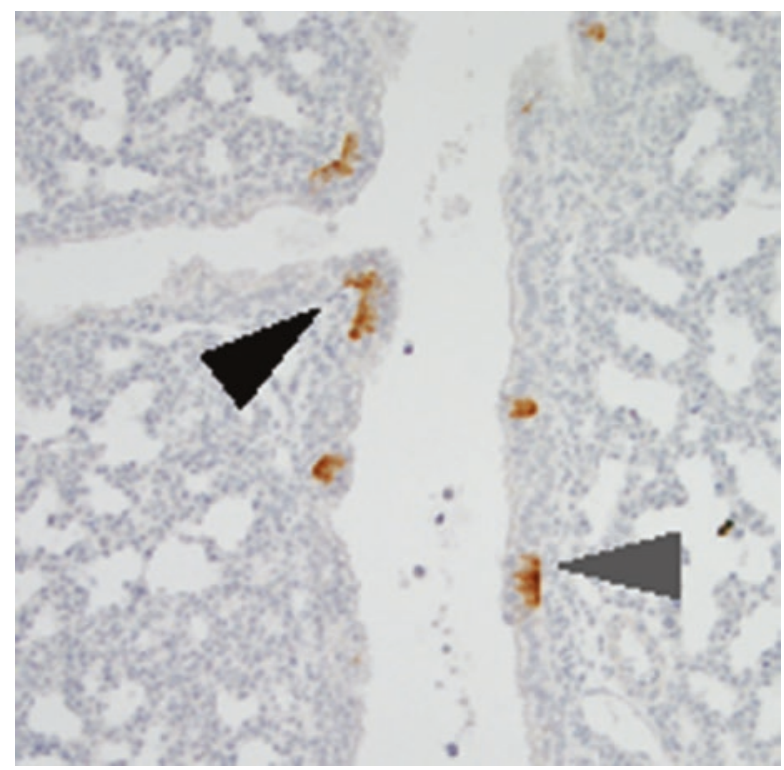

the functional importance of NE cells and NEBs, their developmental course remains unclear in part because of technical limitations in obtaining high-resolution images and quantitative analysis of the behavior of epithelial cells in the context of a 3D branching morphology. We have established methods for 3D mapping of lung epithelial cells and ex vivo 4D imaging of the developing lung [10].

To accurately map the positions of all NEBs throughout the entire respiratory tree, we used two-photon microscopy to image whole-mount lungs in a transgenic mouse strain, $\operatorname{Ret}^{\mathrm{EGFP} /+} ; \mathrm{SH}^{\mathrm{Cre} /+} ; R 26 \mathrm{R}^{\mathrm{H} 2 \mathrm{~B}-\mathrm{mCherry}}$ mice visualizing NE cells in the epithelium $\left(\mathrm{EGFP}^{+} ; \mathrm{mCherry}^{+}\right)$and neuronal cells in the mesenchyme $\left(\mathrm{EGFP}^{+}\right.$; $\mathrm{mCherry}^{-}$). Lungs were collected at E14.5, E15.5, and E16.5 and cleared using clear, unobstructed brain imaging cocktail (CUBIC) solution [11]. The 3D architecture of mCherry ${ }^{+}$lung epithelium was extracted computationally from 3D image stacks by removal of mesenchymal signals, and data on the positions of all epithelial and NE cells were recorded (Fig. 3.5a). These 3D imaging and quantitative analyses revealed that nodal NEBs are significantly larger than internodal NEBs at each stage. We redefined the localization of nodal NEBs by close examination of their positions within the entire geometric architecture of the airway tubes (Fig. 3.5b). These geometric analyses revealed that nodal NEBs are located at stereotypic positions in airway branching structures.

\subsection{Notch-Hes 1 Signaling Is Required for Restricted Differentiation of Solitary NE Cells}

To determine the mechanisms of Notch-mediated NEB development, we genetically ablated Hesl gene, which is the target of Notch signaling and a regulator of NE cell development [12], from endodermal epithelium by generating $\mathrm{SHH}^{\mathrm{Cre} /+}$; 


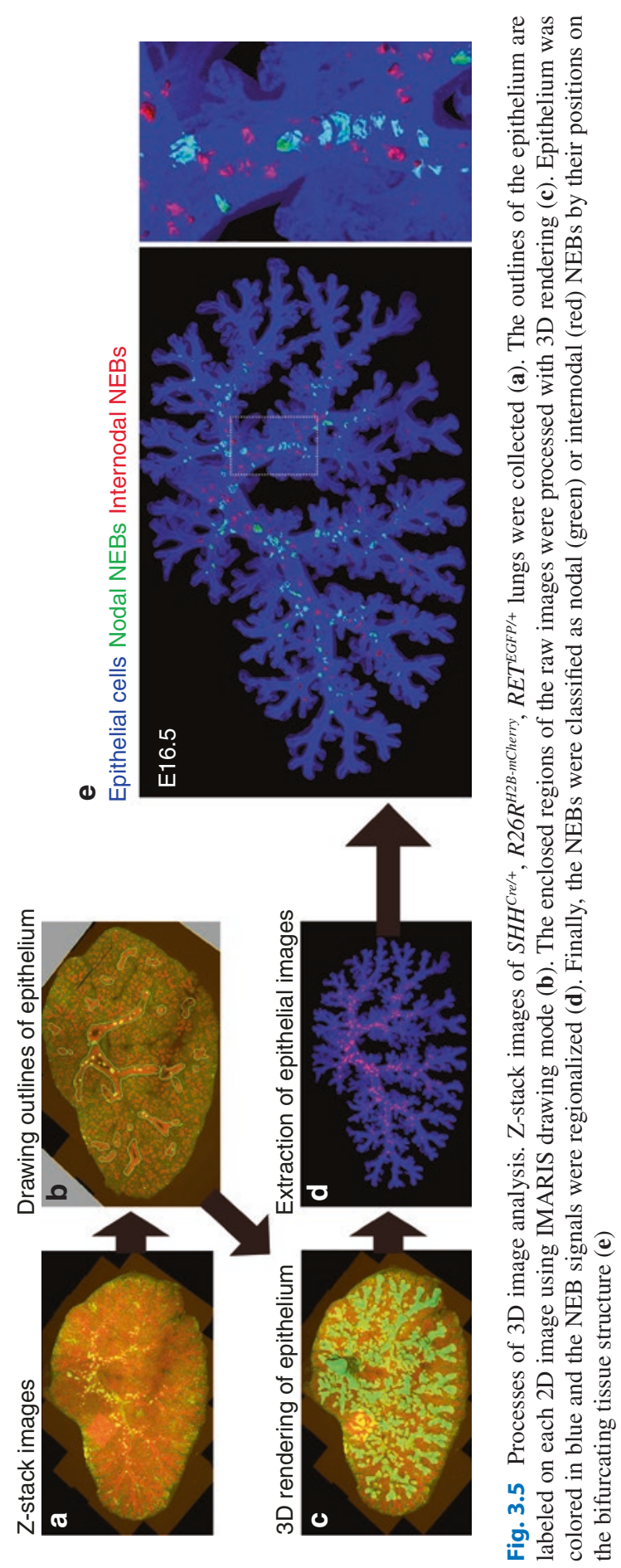


$H e s 1^{\text {f/f }}$ mice. Ascl1-positive primordial NE cells were examined at E13.5. Compared with normal epithelium, the Hes1-deficient epithelium showed abundant NE cells that tended to adjoin each other at the proximal region in Hes1-deficient epithelium (Fig. 3.6a). The punctate distribution of NE cells in the normal E13.5 lung and the disruption of this pattern in Hes1 mutants suggest that Notch-Hes1 signaling suppresses NE cell fate in a classical lateral inhibition fashion (Fig. 3.6b). To analyze the effect of Hes 1 depletion in NEB formation, we performed NEB 3D mapping by generating $\mathrm{SHH}^{\mathrm{Cre} /+} ; \mathrm{R}_{2} 6 \mathrm{R}^{\mathrm{H} 2 \mathrm{~B}-\mathrm{mCherry}} ; \mathrm{Ret}^{\mathrm{EGFP} /+} ; \mathrm{Hes}^{\mathrm{f} / \mathrm{f}}$ embryos and imaged the cranial lobes at E16.5. In contrast to the wide distribution of NE cells at E13.5, we noted markedly enlarged NEBs throughout the proximal to distal airways (Fig. 3.6c).

\subsection{Directional Migration of NE Cells Toward Bifurcation Points Creates Nodal NEBs}

We hypothesized that NE cells emerge as solitary cells and, subsequently, migrate toward a bifurcation point to form NEBs. To test this hypothesis, we established a 4D imaging method for the developing lung, involving 3D plus time-lapse imaging of living tissue. We cultured E13.5 cranial lobes at the air-liquid interface on a membrane filter that becomes transparent in liquid (Fig. 3.7a). In these 4D images, NE cells initially appear as individual cells, in line with our expectations. These cells subsequently migrated toward a bifurcation point located in a more distal region and accumulated there to form an NEB (Fig. 3.7b). Some NE cells also clustered at inter-bifurcation areas. These observations support the idea that NE cells emerge as solitary cells via Notch-mediated cell fate selection and, subsequently, migrate toward bifurcation points on the basement membrane to form nodal NEBs (Fig. 3.8). Internodal NEBs may be generated by a population of cells that are arrested during this migration. In this study, we described a spatial relationship between 3D branching morphology and nodal NEB localization. We also provided direct evidence showing that cell-autonomous Notch-Hes 1 signaling inhibits NE cell differentiation in a classical lateral inhibition fashion, giving rise to a limited number of NE cells. Our results suggest the presence of at least three factors (or combinations of factors) that control NE cell clustering, working to attract them to the distal trap at bifurcation points and induce their aggregation [10]. We are now investigating molecular mechanisms explaining directional migration and clustering at the bifurcating area of the developing airways.

Given that NE cells are thought to be the cells of origin in highly malignant small-cell lung cancers, investigating the molecular mechanisms of NE cell migration may provide important clues toward the development of new therapeutic approaches to mitigate this malignancy.

Acknowledgments Research in the Morimoto lab is supported by Grants-in-Aid for Scientific Research (B) and Young Scientists (A) 26713029 and 23689044 of the Ministry of Education, Culture, Sports, Science and Technology, Japan, and The Takeda Science Foundation for the Life Science (M.M.). 


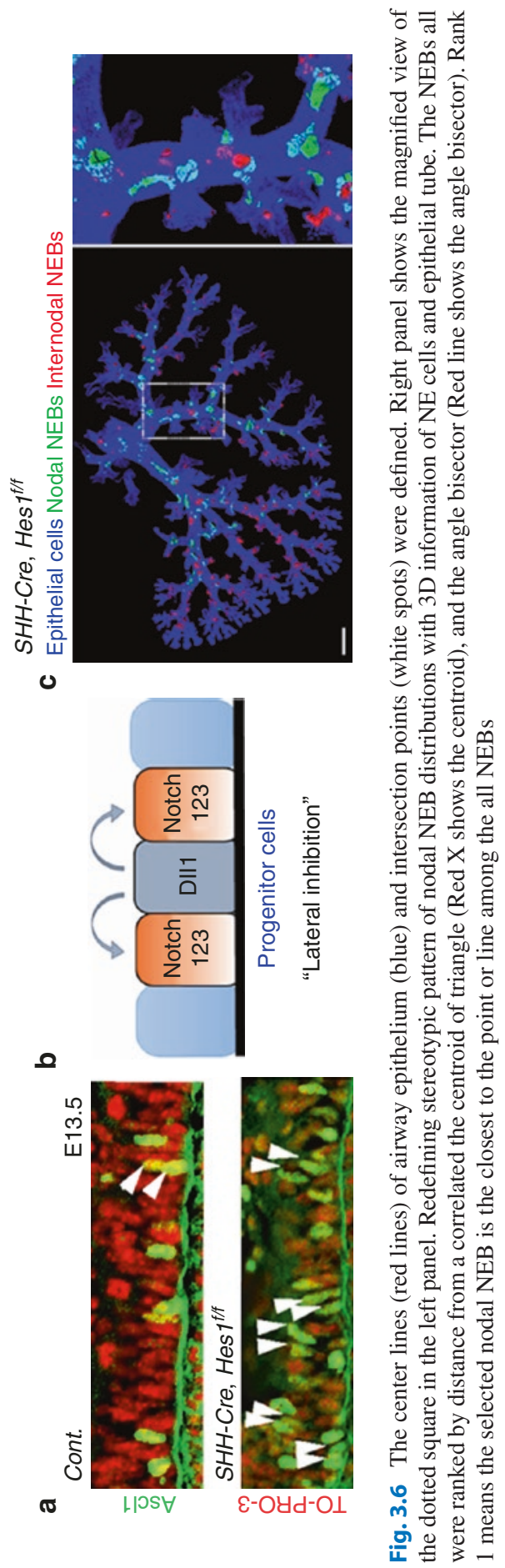




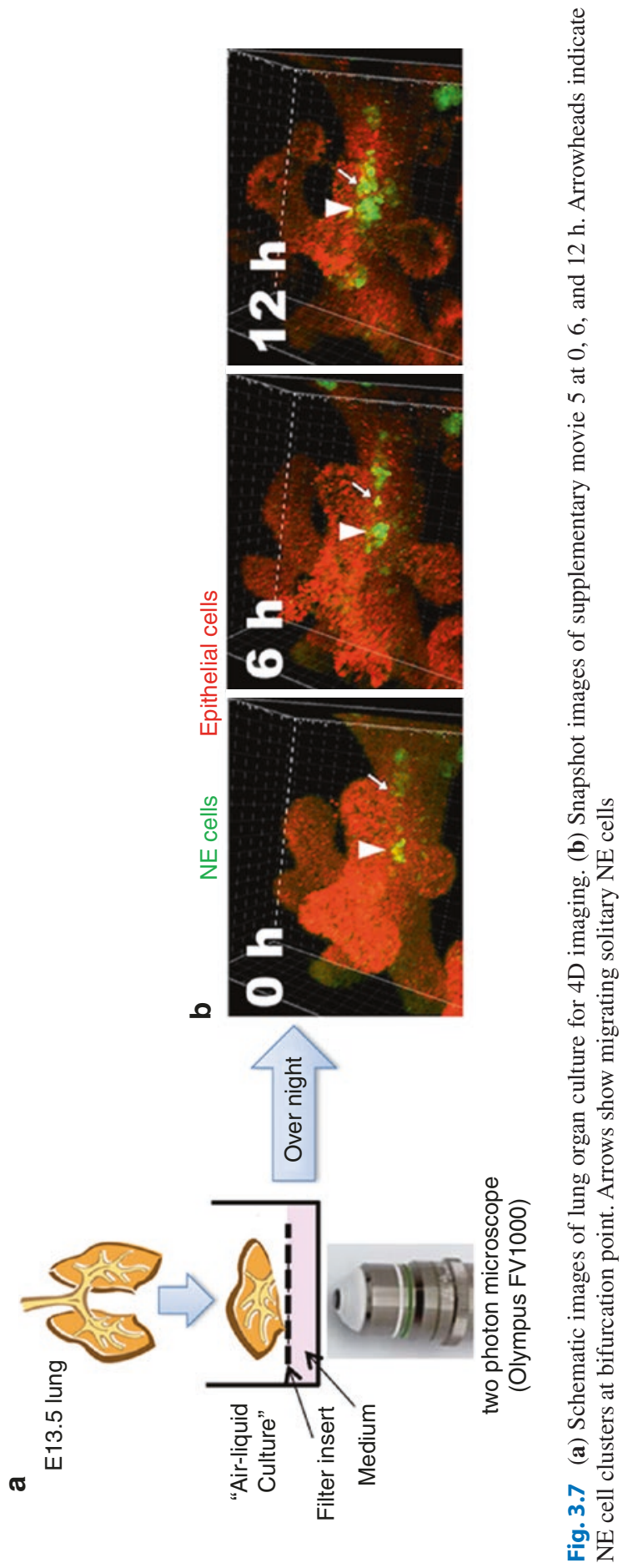


Fig. 3.8 Conclusion

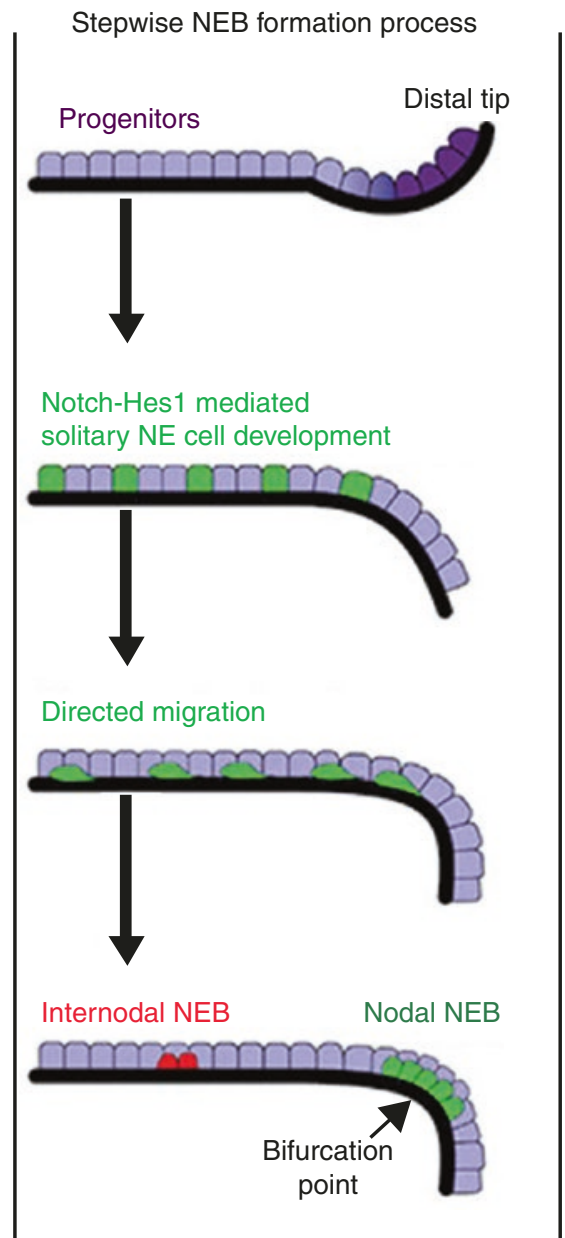

\section{References}

1. Hogan BL, Barkauskas CE, Chapman HA, et al. Repair and regeneration of the respiratory system: complexity, plasticity, and mechanisms of lung stem cell function. Cell Stem Cell. 2014;15(2):123-38.

2. Morrisey EE, Hogan BL. Preparing for the first breath: genetic and cellular mechanisms in lung development. Dev Cell. 2010;18(1):8-23.

3. Perl AK, Wert SE, Nagy A, et al. Early restriction of peripheral and proximal cell lineages during formation of the lung. Proc Natl Acad Sci U S A. 2002;99(16):10482-7.

4. Metzger RJ, Klein OD, Martin GR, et al. The branching programme of mouse lung development. Nature. 2008;453(7196):745-50.

5. Rawlins EL, Clark CP, Xue Y, et al. The Id2+ distal tip lung epithelium contains individual multipotent embryonic progenitor cells. Development. 2009;136(22):3741-5.

6. Cardoso WV, Lu J. Regulation of early lung morphogenesis: questions, facts and controversies. Development. 2006;133(9):1611-24. 
7. Morimoto M, Liu Z, Cheng HT, et al. Canonical notch signaling in the developing lung is required for determination of arterial smooth muscle cells and selection of Clara versus ciliated cell fate. J Cell Sci. 2010;123(Pt 2):213-24.

8. Morimoto M, Nishinakamura R, Saga Y, et al. Different assemblies of notch receptors coordinate the distribution of the major bronchial Clara, ciliated and neuroendocrine cells. Development. 2012;139(23):4365-73.

9. Cutz E, Chan W, Sonstegard KS. Identification of neuro-epithelial bodies in rabbit fetal lungs by scanning electron microscopy: a correlative light, transmission and scanning electron microscopic study. Anat Rec. 1978;192(3):459-66.

10. Noguchi M, Sumiyama K, Morimoto M. Directed migration of pulmonary neuroendocrine cells toward airway branches organizes the stereotypic location of neuroepithelial bodies. Cell Rep. 2015;13(12):2679-86.

11. Susaki EA, Tainaka K, Perrin D, et al. Whole-brain imaging with single-cell resolution using chemical cocktails and computational analysis. Cell. 2014;157(3):726-39.

12. Ito $\mathrm{T}$, Udaka $\mathrm{N}$, Yazawa $\mathrm{T}$, et al. Basic helix-loop-helix transcription factors regulate the neuroendocrine differentiation of fetal mouse pulmonary epithelium. Development. 2000;127(18):3913-21.

Open Access This chapter is licensed under the terms of the Creative Commons Attribution 4.0 International License (http://creativecommons.org/licenses/by/4.0/), which permits use, sharing, adaptation, distribution and reproduction in any medium or format, as long as you give appropriate credit to the original author(s) and the source, provide a link to the Creative Commons license and indicate if changes were made.

The images or other third party material in this chapter are included in the chapter's Creative Commons license, unless indicated otherwise in a credit line to the material. If material is not included in the chapter's Creative Commons license and your intended use is not permitted by statutory regulation or exceeds the permitted use, you will need to obtain permission directly from the copyright holder.

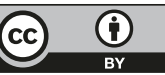

